University of New Hampshire

University of New Hampshire Scholars' Repository

Space Science Center

Institute for the Study of Earth, Oceans, and

Space (EOS)

2006

\title{
Medium-Energy Gamma-Ray Astrophysics with the 3-DTI Gamma- Ray Telescope
}

\author{
Stanley D. Hunter \\ NASA Goddard Space Flight Ctr \\ Robert G. Baker \\ NASA Goddard Space Flight Ctr \\ Louis M. Barbier \\ NASA/Goddard Space Flight Center \\ Peter F. Bloser \\ University of New Hampshire, Peter.Bloser@unh.edu \\ La Vida Cooper \\ NASA Goddard Space Flight Ctr
}

See next page for additional authors

Follow this and additional works at: https://scholars.unh.edu/ssc

Part of the Astrophysics and Astronomy Commons

\section{Recommended Citation}

Hunter, S.D.; Baker, R.G.; Barbier, L.M.; Bloser, P.F.; Cooper, L.; Krizmanic, J.F.; Link, J.T.; McConnell, M.L.; de Nolfo, G.A.; Ryan, J.M.; Singh, S.; Seunghee Son, "Medium-Energy Gamma-Ray Astrophysics with the 3-DTI Gamma-Ray Telescope," Nuclear Science Symposium Conference Record, 2006. IEEE , vol.2, no., pp.778,782, Oct. 29 2006-Nov. 12006

This Conference Proceeding is brought to you for free and open access by the Institute for the Study of Earth, Oceans, and Space (EOS) at University of New Hampshire Scholars' Repository. It has been accepted for inclusion in Space Science Center by an authorized administrator of University of New Hampshire Scholars' Repository. For more information, please contact Scholarly.Communication@unh.edu. 


\section{Authors}

Stanley D. Hunter, Robert G. Baker, Louis M. Barbier, Peter F. Bloser, La Vida Cooper, John F. Krizmanic, Jason T. Link, Mark L. McConnell, Geoergia A. De Nolfo, James M. Ryan, Satpal Singh, and Seunghee Son 


\title{
Medium-Energy Gamma-Ray Astrophysics with the 3-DTI Gamma-Ray Telescope
}

\author{
Stanley D. Hunter, Robert G. Baker, Louis M. Barbier, Peter F. Bloser, LaVida Cooper, John F. Krizmanic, \\ Jason T. Link, Mark L. McConnell, Georgia A. de Nolfo, James M. Ryan, Satpal Singh, and Seunghee Son
}

\begin{abstract}
Gamma-ray observations in the medium energy range (0.50-50.0 MeV) are central to unfolding many outstanding questions in astrophysics. The challenges of medium-energy gamma-ray observations, however, are the low photon statistics and large backgrounds. We review these questions, address the telescope technology requirements, and describe our development of the 3-Dimensional Track Imaging (3-DTI) Compton telescope and its performance for a new mediumenergy gamma-ray mission. The 3-DTI is a large-volume time projection chamber (TPC) with a 2-dimensional gas micro-well detector (MWD) readout.
\end{abstract}

\section{INTRODUCTION}

$\mathrm{T}_{\mathrm{s}}^{\mathrm{h}}$ he first major full-sky survey in medium-energy (0.4 to 30 $\mathrm{MeV}$ ) gamma-ray astrophysics was made with the COMPTEL instrument on the Gamma Ray Observatory from April 5, 1991 to June 4, 2000. The COMPTEL results addressed a broad range of astrophysics including cosmic particle accelerators, nucleosynthesis and cosmic radioactivity, Galactic diffuse emission, and cosmology. The COMPTEL catalog of objects includes Active Galactic Nuclei \& Blazars (Mkn 421, 3C273), Super Nova Remnants (Crab Nebula), Pulsars \& Magnetars (PSR 1509-58), and Black Hole Binaries (Cyg X-1). These observations indicate that we are on the threshold of major discoveries addressing fundamental questions of thermal and relativistic astrophysics in the universe.

Expanding on the COMPTEL science results requires development of a future gamma-ray telescope with greatly improved sensitivity by increasing the overall aperture and effectively addressing background rejection. In addition, this telescope will need to incorporate several measurement techniques since the objects that produce these gamma-rays are both extended $\&$ point-like, transient $\&$ steady, and include both continuum $\&$ line emissions. A study of these techniques has recently been completed for the Advanced Compton Telescope (ACT), one of NASA's future Vision Missions [1].

The ACT report calls for an increase in sensitivity of about 100 times that of COMPTEL. The primary science objective assumed for the ACT study report is to detect the ${ }^{56} \mathrm{Co}(0.847$

Manuscript received November 17, 2006.

S. D. Hunter (telephone: 301-286-7280, e-mail: Stanley.D.Hunter@ nasa.gov), L. M. Barbier, R. G. Baker, LaVida Cooper, \& S. Singh are with NASA/Goddard Space Flight Center, Greenbelt, MD 20771 USA

G. A. de Nolfo, J. F. Krizmanic, and J. T. Link with CRESST/USRA working at NASA/GSFC, Greenbelt, MD 20771 USA

S. Son is a NASA Post Doctoral fellow working at NASA/GSFC, Greenbelt, MD 20771 USA

P. F. Bloser, M. L. McConnell, and J. M. Ryan are with University of New Hampshire, Space Science Center, Durham, NH 03824 USA
$\mathrm{MeV}$ ) line produced in thermonuclear supernovae (SNe Ia), which will shed light on the dynamics of nuclear burning. This narrow science goal was used to define a baseline instrument design. The desired performance of this baseline instrument has an effective area of about $10^{3} \mathrm{~cm}^{2}$, broad-line (3\%) sensitivity of $1.2 \times 10-6 \mathrm{ph} \mathrm{cm}^{-2} \mathrm{~s}^{-1}$ at $0.847 \mathrm{MeV}$, narrow-line sensitivity of $5 \times 10^{-7} \mathrm{ph} \mathrm{cm}^{-2} \mathrm{~s}^{-1}$, and continuum sensitivity of $(1 / \mathrm{E}) \times 10^{-5} \mathrm{ph} \mathrm{cm}^{-2} \mathrm{~s}^{-1} \mathrm{MeV}^{-1}$. This performance represents an increase in narrow- and broad-line sensitivity of about 100-fold over COMPTEL.

The ACT mission is not expected to start before 2012, with a launch date well after the end of the INTEGRAL and GLAST missions. However, between the start of the GLAST mission and the ACT missions, there is a possibility of an intermediate, MIDEX scale, medium-energy gamma-ray mission.

This intermediate mission, with a 10-fold increase in narrow-line, broad-line, and continuum sensitivity over COMPTEL, would bridge the science from COMPTEL to ACT, fill in the multi-wavelength coverage gap between INTEGRAL and GLAST, and substantially advance our scientific understanding of transition between thermal and relativistic processes in the universe.

The science goals enabled by a 10 -fold increase in sensitivity translate into three requirements that drive the design of a medium-energy telescope.

- Address the widest range of science goals by optimizing the sensitivity to both line and continuum emission, to both point-like and extended objects, and to both steady state and transient sources.

- Extend the energy range down to $0.3 \mathrm{MeV}$ and up to 50 $\mathrm{MeV}$ by photon imaging from both Compton scattering and pair production interactions.

- Maximize the effective area by improving the background rejection.

In the following, we describe our efforts towards the design and fabrication of a Compton telescope suitable for this intermediate mission.

\section{COMPton Scattering Telescope Design}

Compton scattering telescopes, Fig. 1, use two separated, position sensitive detectors. An incident gamma ray scatters in the first detector (D1, track imager) producing a recoil electron. The scattered gamma ray is then totally absorbed after one or more interactions in the second detector (D2, calorimeter). Measurement of the position of the single 


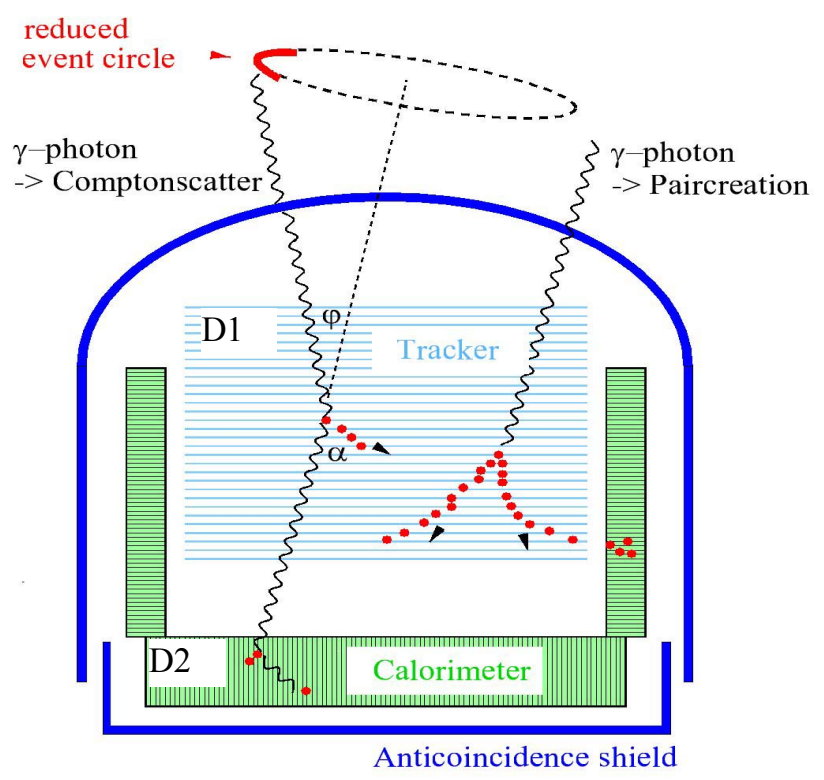

Fig. 1. Schematic of the MEGA medium-energy telescope [2]. The Si-strip D1 track imager provides only a few $(<\sim 20)$ position measures of the recoil electron tracks from Compton scattering or pair production.

Compton scatter and the total energy of the recoil electron in D1, and the position and energy of the scattered gamma ray in D2, is sufficient to determine the energy of the incident gamma ray and the Compton scattering angle $\varphi$, Fig. 2. Thus the point spread function (PSF) of a single event is a circle on the sky called the event circle, Fig. 2a. The MEGA mediumenergy gamma-ray telescope [2], is one concept for a more advanced Compton telescope with tracking of the recoil electron, uses multiple layers of planar X- and Y-position sensitive silicon-strips as the D1 detector, Fig. 1. The MEGA design provides a few $(<20)$ position measurements of the recoil electron track, which constrains the plane of the interactions. Thus the event circle is reduced to an event arc with angular length $\Delta \theta$, Fig. $2 \mathrm{~b}$. The length of the arc is determined by the accuracy with which the initial recoil electron direction can be constrained, which is in turn limited by multiple scattering in the tracker material.

The Compton scatter angle, $\varphi$, and energy of the incident gamma ray can be derived from measurements of the energy of the recoil electron and the scattered gamma ray using the Compton formula. The direction of the incident gamma ray is equally probable from any point on the event circle determined by the scatter angle. Measuring the direction of recoil electron reduces the event circle to an event arc with azimuthal extent, $\Delta \theta$, Fig. $2 \mathrm{~b}$. For a Compton telescope, the background associated with any observed photon is proportional to the area of the event circle, $\sin \varphi \Delta \varphi \Delta \theta$, thus reducing the azimuthal extent of the arc reduces the observed background and hence increases the sensitivity of the telescope. The ability to constrain the incident gamma-ray direction with highly precise imaging techniques and thus
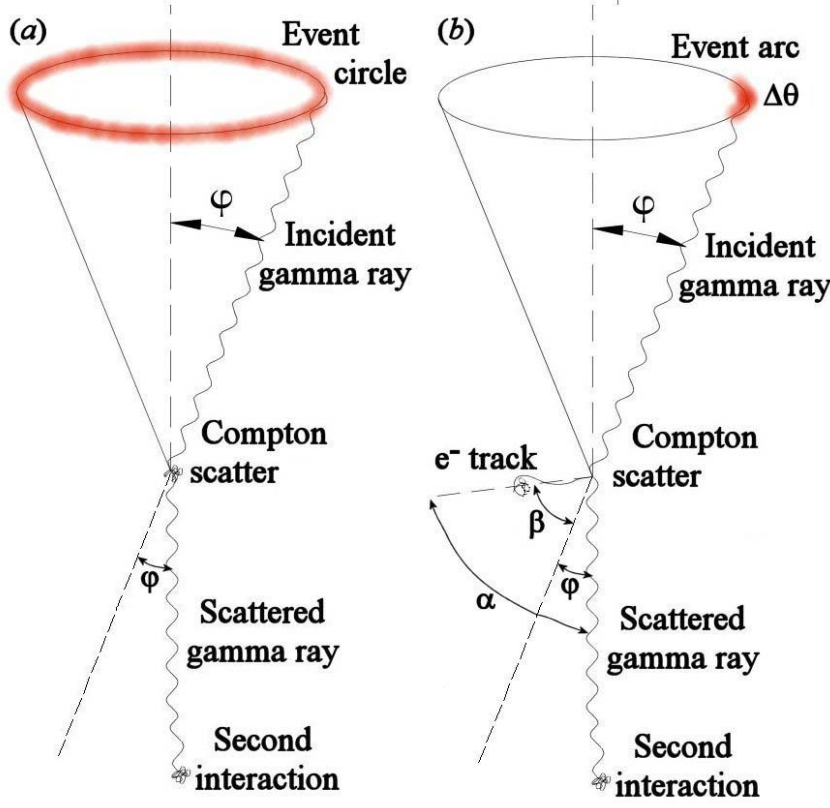

Fig. 2. (a) Compton scattering without electron tracking constrains the incident gamma ray direction on an event circle, (b) Tracking the recoil electron reduces the event circle to an arc. The uncertainty in the electron azimuth determines the arc length.

dramatic improvements in sensitivity opens up the field of medium-energy gamma-ray observations to many exciting new potential discoveries.

The science goals and design drivers of an intermediate Compton telescope mission can be achieved with the MEGA two-detector design with an improved D1 track imager. Towards this goal, we are developing the Three-Dimensional Track Imager (3-DTI) that provides hundreds of track position measurements in a low-density gaseous medium as part of a complete Compton telescope for an intermediate mediumenergy gamma-ray mission.

\section{THE 3-DTI TRACK IMAGER}

The 3-DTI, Fig. 3, is large volume time projection chamber (TPC) with a two-dimensional gas micro-well detector (MWD) readout plane. The 3-DTI is designed to measure the three-dimension momentum of charged particles by high spatial resolution sampling of the charged particle ionization tracks in a low density, isotropic, fully active gas volume. The MWD provides $\mathrm{X}$ - and $\mathrm{Y}$-coordinates and the 3rd dimension is obtained by measuring the drift time of the ionization electrons. We describe the 3-DTI TPC and MWD design, front-end and transient digitizer electronics, segmented calorimeter, and performance in the following sections.

\section{A. TPC and MWD Design}

The micro-well detector is a type of gas proportional counter related to the gas electron multiplier [3]. In contrast to multi-wire proportional counter construction, the anodes and cathodes of all these detectors are rigidly affixed to an 


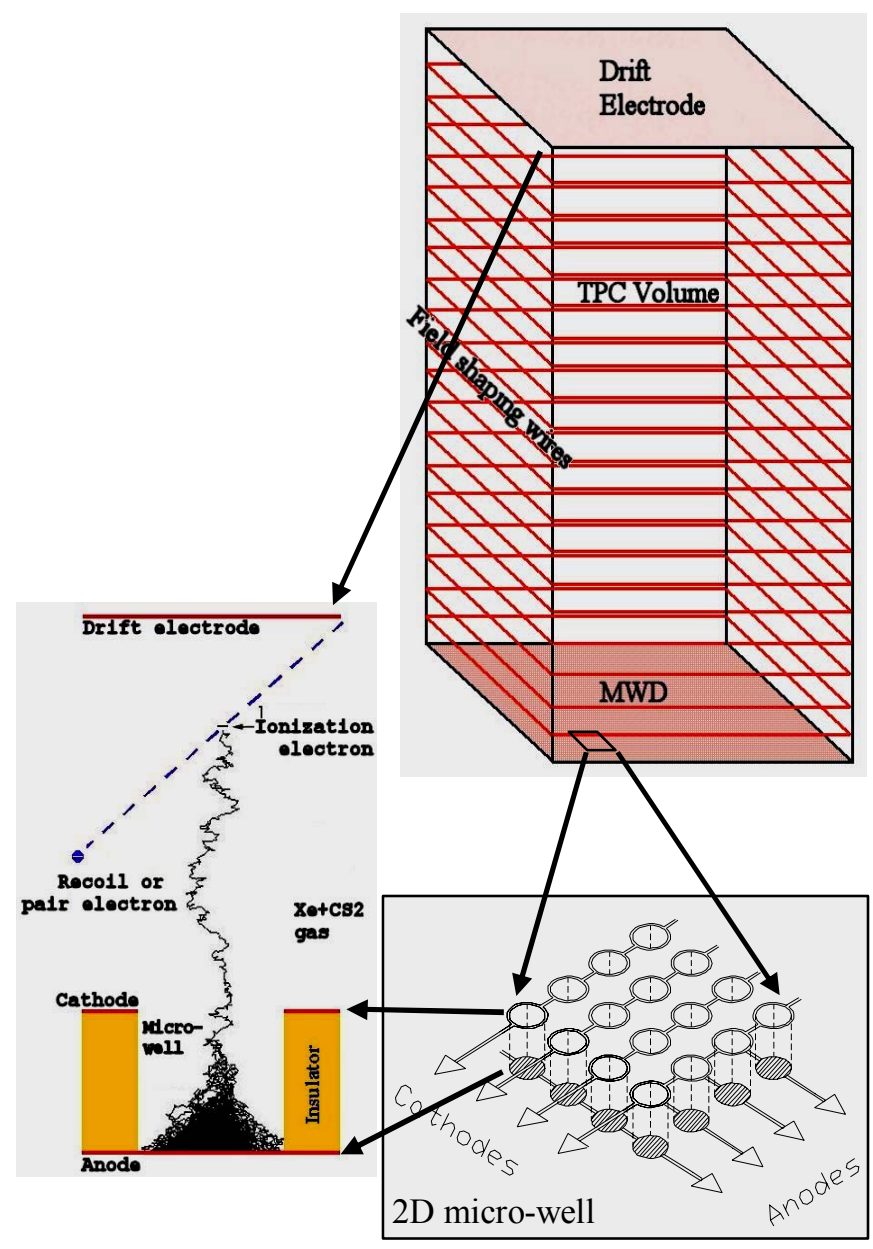

Fig. 3. The 3-DTI is a large volume time projection chamber with 2-D micro-well readout. The ionization charge in the TPC volume drifts towards the MWD and into the individual wells. The strong electric field in the wells produces an avalanche of secondary ionization. The avalanche charge is collected on the anode strip under the well and an equal but opposite mirror charge appears on the cathode.

insulating substrate, Fig. 4. This greatly simplifies their construction and allows the anode and cathode pitches to be smaller than is possible with multi-wire construction. This geometry is also inherently two-dimensional; the anodes give one coordinate and the cathodes the other.

An array of wells forms a detector. The active tracking volume is bounded by a drift electrode on the top and an array of wells on the bottom. Ionization electrons produced as a charged particle looses energy traversing the gas drift toward the anode into the wells of the detector. An ionization avalanche occurs in the well, where there is an intense electric field set up by the voltage difference applied between the anode and cathode. The micro-wells serve as the proportional counter where the electric field strength is large enough to produce secondary ionization of gas atoms. The ensuing avalanche of electrons collects on the anode while charge of equal magnitude but opposite sign collects on the cathode.

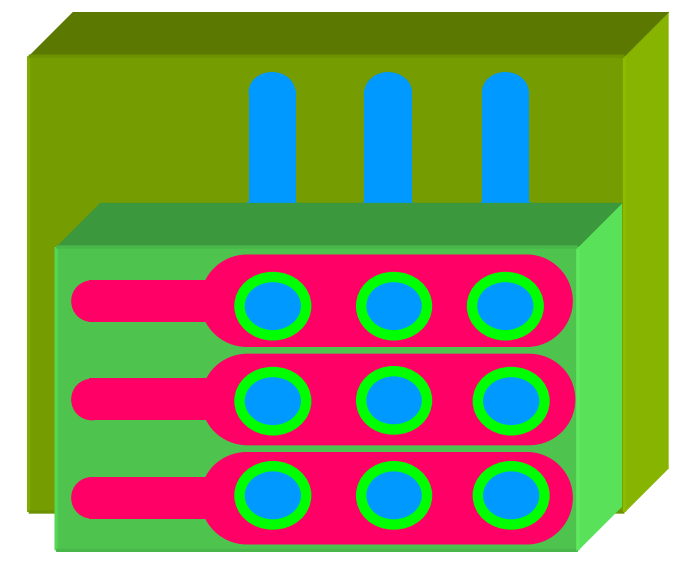

Fig. 4. Schematic view of a micro-well detector. The anodes, blue, are separated from the cathodes, red, by an insulating layer, light green. The MWD is mounted on a rigid substrate, dark green.

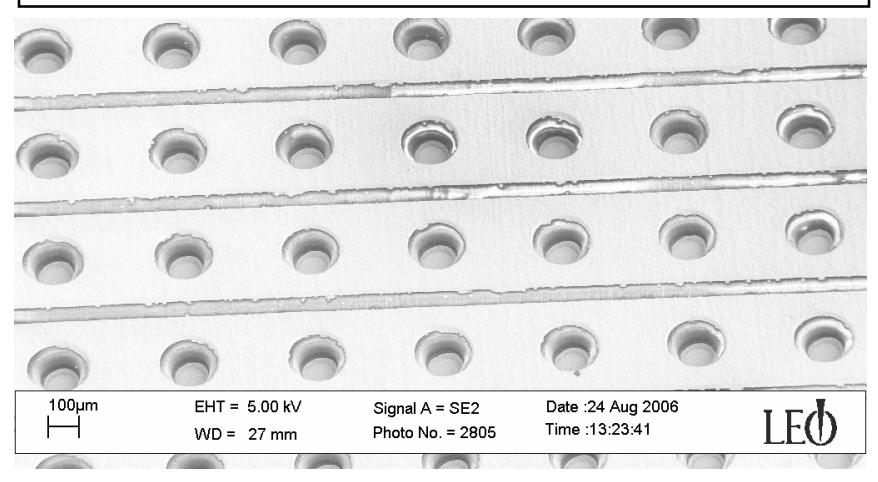

Fig. 5. SEM Micrograph of a micro-well detector. The wells and anode strips can be seen through the holes in the cathode strips

\section{1) $\mathrm{TPC}$}

The TPC and MWD are housed in a pressure vessel which serving as the TPC chamber. Laboratory testing is being done with P-10 (90\% argon, 10\% methane) nominally at 760 Torr. The TPC chamber assembly includes a drift electrode held at 1 $\mathrm{kV}$ and employs a field-shaping grid along the chamber.

2) $M W D$

We have previously constructed two-dimensional crossedstrip MWDs and demonstrated gas gain up to 30,000 and spatial resolution of $\sim 200 \mu \mathrm{m}$ [5]. We are currently exploring ways to produce MWDs that are suitable for commercial fabrication of detectors up to $50 \times 50 \mathrm{~cm}^{2}$ in size. MWDs are laminated substrates where the laminate thickness determines the well thickness and thus the gain. Our MWDs consist of 5 $\mu \mathrm{m}$ copper foil on laminate substrates such as Vacrel and Kapton, Fig. 5. The electrode pattern is a photochemical process. We are considering several different methods for the micro-well machining such as a photochemical etch or laser ablation which are ideal for large area production with relatively little process contamination. Both $5 \times 5 \mathrm{~cm}^{2}$ and $10 \times 10 \mathrm{~cm}^{2}$ MWDs have been produced. The wells have a diameter and depth of $200 \mu \mathrm{m}$ with a pitch of $400 \mu \mathrm{m}$ and a gap between the electrodes of $5 \mu \mathrm{m}$, resulting in a total of 448 micro-wells. 


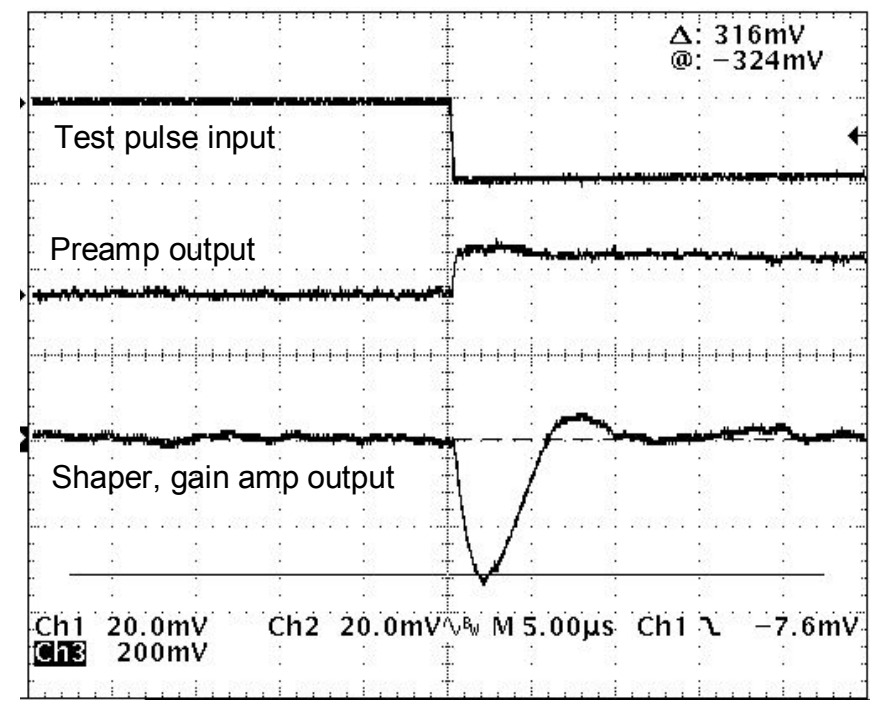

Fig. 6. Response of front-end electronics (FEE) to a test input pulse across a $2 \mathrm{pF}$ capacitor. The overall gain is about $8 \mathrm{mV} / \mathrm{fC}$.

\section{3) Front-end Electronics and Transient Digitizer}

Each anode and cathode electrode of the MWD is connected to a front-end electronics (FEE) channel consisting of a charge sensitive preamplifier, a CR-RC pulse shaper with gain, and a differential driver. The FEE is a bipolar design to accommodate the negative anode signal and positive, cathode mirror signal. To maximize design flexibility, we have chosen to implement the FEE design using discrete components. This initial approach greatly reduced the time required for the design and testing, and permits in-house fabrication. The components used are the AD8610, AD8672 and AD8137 for the charge sensitive preamplifier, the shaping and gain amps, and the driver, respectively. Sixteen FEE channels were fabricated on each front-end card (FEC) which also has the high voltage distribution buss. Our data acquisition is done in LabView. The response of each stage of the FEE to a test pulse input is shown in Fig. 6.

The arrival time of charge pulses from each FEE channel is recorded with a transient digitizer. Since the ionization charge drifts at a constant velocity, the relative pulse arrival time provides the third coordinate of the charge position. In order to achieve equal resolution in all three spatial coordinates, the timing resolution must be equal to the pixel pitch divided by the electron drift velocity. To satisfy the above condition, a 2.5 $\mathrm{MHz}, 12$ bit resolution custom Transient Digitizer (TD) board was designed and has been fabricated. The digitized positive and negative output from an anode and cathode is shown in Fig. 7.

\section{B. Calorimeter (D2 Detector)}

Surrounding the 3-DTI on all four sides and along the bottom will be a segmented calorimeter with an inner layer of $\mathrm{LaBr}_{3}$ crystals and an outer layer of NaI crystals. Surrounding the calorimeter will be anti-coincidence scintillator. We are currently testing, simulating, and optimizing the configuration

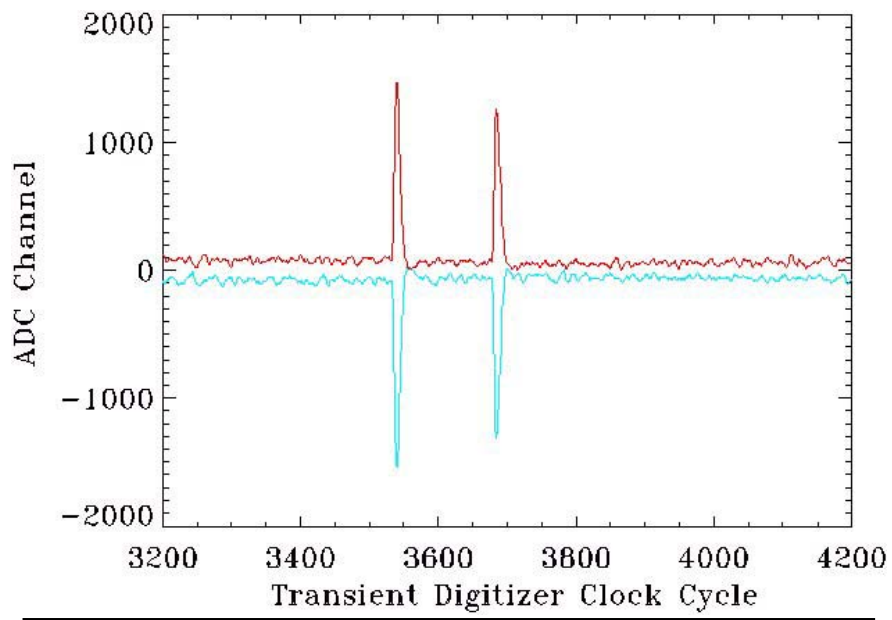

Fig. 7. Digitized positive and negative outputs from an anode and cathode in response to two separate ${ }^{55} \mathrm{Fe}$ events.

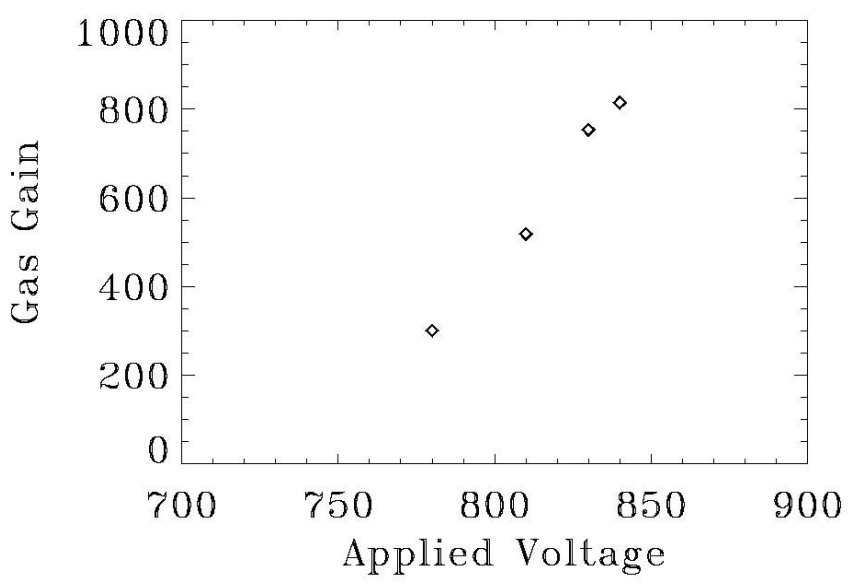

Fig. 8. Micro-well detector (MWD) gas gain (avalanche charge multiplication) measured in P-10 (90\% $\left.\mathrm{Ar}, 10 \% \mathrm{CH}_{4}\right)$ as a function of anode-cathode voltage.

of scintillation crystals to provide the needed energy and position resolution, and to enable a viable, affordable detector.

\section{Instrument Performance}

We tested the 3-DTI prototype in the laboratory using an ${ }^{55} \mathrm{Fe}$ source. Figure 4 shows the energy spectrum for $5.9 \mathrm{keV}$ $\mathrm{X}$-rays with a secondary peak from the Ar escape peak at 2.7 $\mathrm{keV}$. Our energy resolution is $\sim 20 \%$ based on the $5.9 \mathrm{keV} \mathrm{x}-$ ray peak in $90 \%$ argon with $10 \%$ methane at a gain of $10^{3}$.

Gas-gain measurements have been made with a similar micro-well configuration [4] and exhibit stable operation at gas-gains in excess of 30,000. Fig. 8 shows the avalanche or gas gain versus high voltage curves for a $200 \mu \mathrm{m}$ thick MWD. The maximum gain observed to date is $\sim 10^{3}$. The energy resolution of this MWD is shown in Fig. 9 for a voltage difference between the anode and cathode of $1000 \mathrm{~V}$ and. The charge collected on the MWDs is sent to front-end electronics boards (FEE) for charge-integrating amplification and shaping and to transient digitizer boards (TD) for time projection determination. 


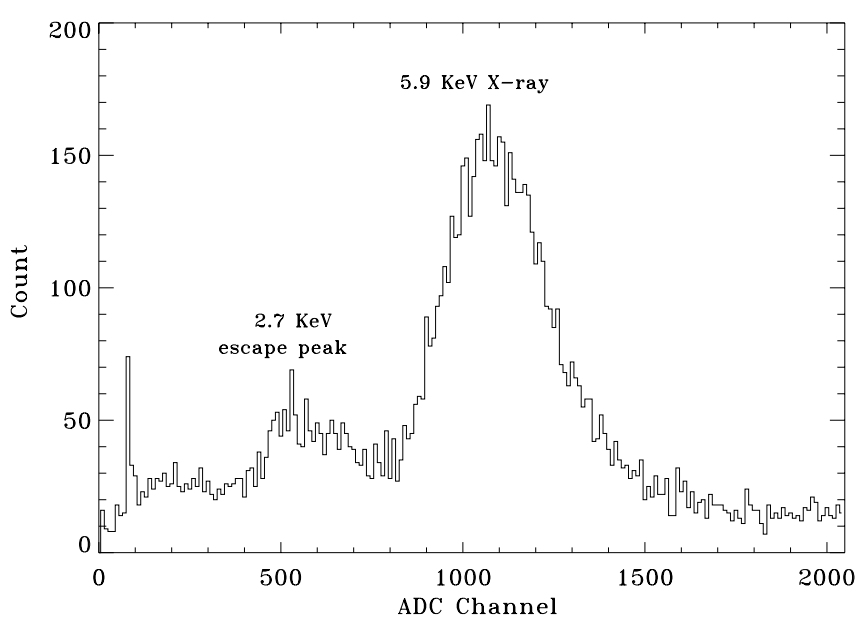

Fig. 9. Measured spectral response of a MWD to 55Fe. The 6 $\mathrm{keV}$ absorption peak and the $3 \mathrm{keV}$ Argon escape peak are clearly visible. The energy resolution is about $20 \%$.

\section{Development Plans}

Arrangements have been made to test a $10 \mathrm{~cm}^{2} 3$-DTI at the 3 MV Tandem Ion Accelerator located at the Naval Surface Warfare Center - Carderock Division in Bethesda Maryland. This single-user facility accelerator allows for the production of mono-energetic gamma-rays between $0.2-15 \mathrm{MeV}$ by making an appropriate selection of ion, target and ion energy. The ability to generate a mono-energetic gamma-ray beam will allow us to calibrate and characterize the 3-DTI detector tracking and verify simulation results. The accelerator testing will also enable the determination of the optimal calorimeter configuration.

\section{CONCLUSIONS}

The MWD fabrication process and the 3-DTI design are being developed with the goal of producing $50 \times 50 \mathrm{~cm}^{2}$ or larger MWDs without extensive retooling. Upon the conclusion of the $10 \times 10 \mathrm{~cm}^{2}$ accelerator tests we will begin the design and fabricate a complete $50 \times 50 \mathrm{~cm}^{2}$ 3-DTI prototype Compton telescope including a complete calorimeter and anti-coincidence. We are planning to fly this prototype onboard a NASA high altitude balloon in 2008.

\section{REFERENCES}

[1] S. Boggs, et al. ACT, Advanced Compton Telescope, NASA Vision Mission Concept Study Report," December 2005

[2] G. Kanbach, et al. "The MEGA project," New Astron. Rev. 48, 275 2004

[3] F. Sauli “,” NIM-A, 386, 531, 1997

[4] P. Deines-Jones et al. "," NIM-A, 477, 55, 2002

[5] P. Deines-Jones et al. “,” NIM-A, 478, 130, 2002 